\title{
Progesterone hypersensitivity: Case report with favorable evolution
}

\author{
SIMONA C. SENILA ${ }^{1 *}$, LOREDANA UNGUREANU $^{1 *}$, ELISABETA CANDREA $^{1}$, SORINA DANESCU $^{1}$, \\ ALINA VASILOVICI ${ }^{1}$, RODICA COSGAREA ${ }^{1}$ and VLAD DIONISIE ${ }^{2}$ \\ ${ }^{1}$ Department of Dermatology, University of Medicine and Pharmacy 'Iuliu Hatieganu', 400006 Cluj-Napoca; \\ ${ }^{2}$ Department of Psychiatry, Clinical Hospital of Psychiatry 'Al. Obregia', 041914 Bucharest, Romania
}

Received July 03, 2018; Accepted August 14, 2018

DOI: 10.3892/etm.2018.7055

\begin{abstract}
Progesterone hypersensitivity or autoimmune progesterone dermatitis is characterized by heterogeneous skin eruptions that cyclically aggravate during the second half of the menstrual cycle, corresponding to a rise in the progesterone level. Clinical presentation is highly variable and includes all urticaria manifestations with or without angioedema, vesiculobullous, eczematous, purpuric or target-like lesions on the skin and mucous membrane. Both endogenous progesterone as well as exogenous progestogens may represent an initial trigger. We report a case of progesterone hypersensitivity in a 27-year old woman with favorable evolution only on topical therapy, the positive clinical outcome being maintained during a subsequent pregnancy and postpartum period.
\end{abstract}

\section{Case report}

A 27-year old Caucasian woman presented herself at the Department of Dermatology for a recurrent pruritic skin eruption during the last 3 months. The patient was breastfeeding for the last 10 months a healthy baby that was born in normal vaginal delivery. The patient recalls having used oral contraceptives as a preventive birth control method for 2 to 5 years prior to the conception that occurred naturally, without need of hormonal treatment or in vitro fertilization.

The cutaneous examination revealed well demarcated, round and oval shaped erythemato-edematous papules and plaques on the cheeks, chin, neck, upper and lower limbs, as well as some target-like lesions, ranging from 5 to $20 \mathrm{~mm}$ (Fig. 1). The patient reported a sudden onset of the skin eruption 3 months prior to the medical visit, starting

Correspondence to: Dr Elisabeta Candrea, Department of Dermatology, University of Medicine and Pharmacy 'Iuliu Hatieganu', 3-5 Clinicilor Street, 400006 Cluj-Napoca, Romania

E-mail: elisabeta.candrea@umfcluj.ro

${ }^{*}$ Contributed equally

Key words: progesterone hypersensitivity, treatment, pregnancy a few days before the menses and lasting until after the completion of menstruation, with clinical worsening at every episode.

A 4-mm punch biopsy was performed and revealed on the histopathological examination spongiosis, a perivascular and diffuse lymphocytic infiltrate in the dermis, a few eosinophils and apoptosis of the basal cells.

Intradermal testing with $0.01 \mathrm{ml}$ progesterone $(50 \mathrm{mg} / \mathrm{ml}$ progesterone at a dilution of 1:10 in aqueous solution) read at $20 \mathrm{~min}$ was positive, showing a $5 \mathrm{~mm}$ wheal, compared to no skin reaction to the saline solution control.

The patient was advised to take oral contraceptives and mid-potency topical corticosteroids but chose to apply only topical treatment. Two months later, the patient became pregnant and delivered a healthy baby on term. The skin eruptions ceased during the second pregnancy and did not reappear at a 1 year follow-up postpartum $(1,2)$.

\section{Discussion}

Progesterone hypersensitivity or autoimmune progesterone dermatitis is a rare disorder that has been scarcely, but worldwide reported. Initially, most cases were associated with exogenous progesterone intake (3); on an extensive review of published data, only $44.95 \%$ of patients recalled exogenous progesterone exposure, however, there was a lack of recorded history for $35.96 \%$ of patients (4). Onset of the disease was also noted in relation to pregnancy, intrapartum, postpartum or after medical abortion (5).

Diagnosis is based on cutaneous eruptions related to increase in progesterone levels and may be confirmed by intracutaneous progesterone testing or progesterone-specific IgE serum. Other available but less useful diagnostic methods include: intramuscular progesterone injection, oral progesterone intake, patch testing (with a low negative predictive value), in vitro leukocyte histamine release assay and interferon-gamma-release assay (6). Clinical presentation is highly variable and includes all urticaria manifestations with or without angioedema, vesiculobullous, eczematous or purpuric lesions, as well as cutaneous eruptions resembling Stevens-Johnson syndrome, erythema multiforme or fixed drug eruptions (4); asthma and anaphylaxis may rarely accompany the symptoms (7). 


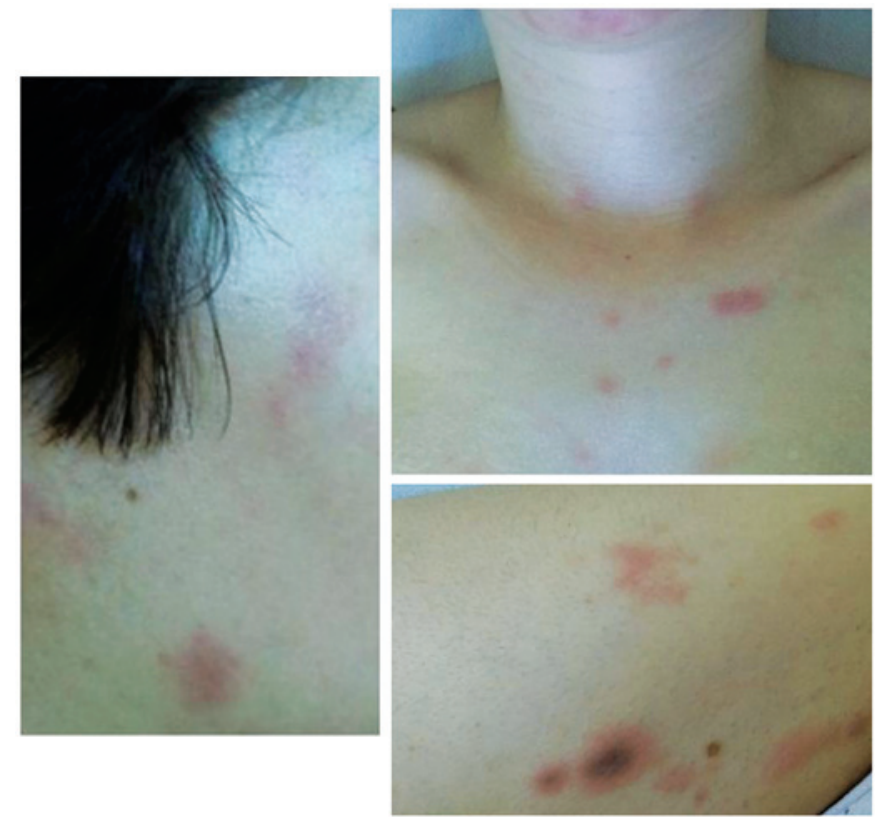

Figure 1. Cutaneous manifestations of progesterone hypersensitivity: Erythemato-edematous papules and plaques, target-like lesions accompanied by pruritus, localized on the lower limbs, presternal area and face.

A review of clinical treatment outcomes was published and reviewed in 2015, summarizing the published data at that time. It found that out of 86 patients with recorded clinical progression, one patient had achieved disease control without treatment and only 4 patients $(4.65 \%)$ achieved complete remission without therapy. Of those who received treatment, complete remission was achieved primarily through total abdominal hysterectomy/bilateral salpingo-oophorectomy (14 cases, representing $16.27 \%$ of all patients and $51.85 \%$ of all patients with complete remission). Medical treatments such as antihistamines, systemic corticosteroids, conjugated estrogen/ethinyl estradiol, gonadotropin releasing hormone, oral contraceptive were mostly associated with disease control or remission (4). Progesterone desensitization was employed in 6 patients, resulting in complete remission in 4, clinical recurrence in one case and disease control in another patient. Oral and intramuscular desensitization protocols were further reported in a retrospective case-series of 11 patients, showing dermatologic symptom improvement in $88 \%$ of cases and cessation of asthma/anaphylactic symptoms (7). The remaining 13 patients, underwent hormone therapy; $62 \%$ of this group also used additional treatments such as antihistamines, topical steroids, montelukast for symptom management. Efficacy of hormonal therapy should mostly be dose and product dependent, however, it is not always true. A 39 year-old multiparous woman diagnosed with autoimmune progesterone dermatitis underwent treatment with vaginal hormonal contraceptives which exacerbated the skin lesions, while oral contraceptive induced a complete symptom resolution (8).

In the present case, we were pleasantly surprised by the evolution of the patient: after onset, the cyclical clinical lesions gradually worsened (showing apoptosis of basal cells on the histopathological examination) but were kept under control with topical corticosteroids. During pregnancy, there is a natural inhibition of ovulation which explains disease remission, however, symptoms may later recur. Fortunately, in this case, the patient presented no disease relapse at a 2 year follow-up from the first presentation. Symptom resolution without treatment has previously been recorded (9), as has minimal symptomatology of no concern to the patient (10). Pregnancy was reported to be associated with symptom release in women diagnosed with autoimmune progesterone dermatitis, possibly due to a gradual increase in progesterone levels (11) but also with clinical exacerbation, even anaphylactic shock during delivery (12).

Patients already diagnosed with progesterone hypersensitivity benefit from more information and better surveillance during pregnancy while asymptomatic women might manifest atypical symptoms. For example, unrevealing workup for habitual idiopathic pregnancy losses showed immediate hypersensitivity towards estrogen and progesterone in $50 \%$ of patients and delayed hypersensitivity in $70 \%$ of patients (13). Even though most women demonstrated combined hypersensitivity, some had only estrogen or progesterone hypersensitivity. A follow-up of women with recurrent pregnancy loss and sex-hormone hypersensitivity that underwent desensitization protocols demonstrated 5 conceptions before desensitization and 16 (out of 26-61\%) conceptions and live births (14).

The above-mentioned studies shift our focus from the typical patient who has cyclical skin eruptions during the luteal phase to women who do not have clinical onset of disease but other symptoms, for example habitual pregnancy losses. From this point of view, a recent report illustrates the case of a 31 year old woman with recurrent pregnancy loss who also developed self limited urticarial or eczematous eruptions in the time-frame corresponding to higher progesterone levels. Following a positive intradermal test to progesterone she underwent desensitization and successfully sustained pregnancy with progesterone supplementation (15).

Unfortunately, not all patients had such a favorable outcome. A recent article describes the case of a 22-year old woman with progesterone hypersensitivity who had to undergo bilateral salpingo-oophorectomy due to unsatisfactory response to various medical treatments including antihistamines, oral contraceptives, corticosteroids, leuprolide acetate and estradiol, tamoxifen and danazol, desensitization protocol, immunomodulators for a period of 48 months (16).

In conclusion, our report addresses the various treatment outcomes of women with progesterone hypersensitivity and highlights the possibility of resolution in the absence of a medical or surgical treatment.

\section{Acknowledgements}

Not applicable.

\section{Funding}

No funding was received.

\section{Availability of data and materials}

The datasets used and/or analyzed during the current study are available from the corresponding author on reasonable request. 


\section{Authors' contributions}

SCS, LU, EC were responsible for the data analysis and interpretation and contributed to writing the manuscript. SAD, AV, VD were responsible for the data selection and clinical interpretation of the data. RC designed the study and interpreted the data. All authors read and approved the final manuscript. All authors read and approved the final manuscript.

\section{Ethics approval and consent to participate}

The study was approved by the Ethics Committee of the Medical Clinic (Cluj-Napoca, Romania), and written consent was given by the patient.

\section{Patient consent for publication}

The patient gave written consent, however, the authors made efforts to remove identifying information to protect the privacy of the patient.

\section{Competing interests}

The authors declare that they have no competing interests.

\section{References}

1. Gheorghe I, Tatu AL, Lupu I, Thamer O, Cotar AI, Pircalabioru GG, Popa M, Cristea VC, Lazar V and Chifiriuc MC: Molecular characterization of virulence and resistance features in Staphylococcus aureus clinical strains isolated from cutaneous lesions in patients with drug adverse reactions. Rom Biotechnol Lett 22: 12321-12327, 2017.

2. Raţiu MP, Purcărea I, Popa F, Purcărea VL, Purcărea TV, Lupuleasa D and Boda D: Escaping the economic turn down through performing employees, creative leaders and growth driver capabilities in the Romanian pharmaceutical industry. Farmacia 59: 119-130, 2011.

3. Hart R: Autoimmune progesterone dermatitis. Arch Dermatol 113: 426-430, 1977.
4. Nguyen T and Razzaque Ahmed A: Autoimmune progesterone dermatitis: Update and insights. Autoimmun Rev 15: 191-197, 2016.

5. Mbonile L: Autoimmune progesterone dermatitis: Case report with history of urticaria, petechiae and palpable pinpoint purpura triggered by medical abortion. S Afr Med J 106: 48-50, 2016.

6. Li RC, Buchheit KM and Bernstein JA: Progestogen hypersensitivity. Curr Allergy Asthma Rep 18: 1, 2018.

7. Foer D, Buchheit KM, Gargiulo AR, Lynch DM, Castells M and Wickner PG: Progestogen hypersensitivity in 24 Cases: Diagnosis, management, and proposed renaming and classification. J Allergy Clin Immunol Pract 4: 723-729, 2016.

8. Camões S, Sampaio J, Rocha J, Tiago P and Lopes C: Autoimmune progesterone dermatitis: Case report of an unexpected treatment reaction. Australas J Dermatol 58: e132-e134, 2017.

9. Herzberg AJ, Strohmeyer CR and Cirillo-Hyland VA: Autoimmune progesterone dermatitis. J Am Acad Dermatol 32: 333-338, 1995.

10. Özmen İ and Aktürk E: Autoimmune progesterone dermatitis presenting with purpura. Cutis 98: E12-E13, 2016.

11. García-Ortega P and Scorza E: Progesterone autoimmune dermatitis with positive autologous serum skin test result. Obstet Gynecol 117: 495-498, 2011.

12. O'Rourke J,Khawaja N,Loughrey J and McKenna P: Autoimmune progesterone dermatitis in a parturient for emergency caesarean section. Int J Obstet Anesth 13: 275-278, 2004.

13. Untersmayr E, Jensen AN and Walch K: Sex hormone allergy: Clinical aspects, causes and therapeutic strategies - Update and secondary publication. World Allergy Organ J 10: 45, 2017.

14. Itsekson AM, Soriano D, Zolti M, Seidman DS and Carp HJ: Intradermal sex hormone desensitization for relief of premenstrual symptoms may improve the obstetric outcome of women with recurrent pregnancy loss. Gynecol Endocrinol 29: 169-172, 2013.

15. Kuruvilla M, Vanijcharoenkarn K, Wan J, Pereira N and Chung P: Exogenous progesterone hypersensitivity associated with recurrent pregnancy loss J Allergy Clin Immunol Pract 6: 1412-1413, 2018.

16. Drayer SM, Laufer LR and Farrell ME: Autoimmune progesterone dermatitis presenting as Stevens-Johnson Syndrome. Obstet Gynecol 130: 881-884, 2017.

This work is licensed under a Creative Commons Attribution-NonCommercial-NoDerivatives 4.0 International (CC BY-NC-ND 4.0) License. 